\title{
Bone augmentations with autologous bone for reconstruction 3D in oral implantology
}

\author{
Vîrbănescu CA*, Horia B and Referendaru D \\ Faculty of dental medicine, University titu maiorescu, Bucharest, Romania
}

\section{Introduction}

Oral implantology is a branch of surgery that opened a new era in stomatology and whose unpredictable development we cannot predict accurately. We can say that oral implantology is a mixture of dentoalveolar, prosthetic and gnathology surgery. The findings and the full content of oral implantology are amplified and shaped at a very alert pace.

Oral implantology has led to the change of principles and overturned general concepts of dentistry. That is why in 1996 in Bucharest, Iasi, Cluj, and Timisoara it became a mandatory discipline.

The concept of osteointegration, guided tissue regeneration, bone grafts, sinus-lift interventions has contributed to expanding dental implant indications and increasing the success rate.

In the therapy of bone defects for the purpose of substituting the hard tissues, additive materials are used.

These in the form of organic are divided into autographs, analogs, and alloplastic materials.

Since ancient times, the lost teeth have been replaced, the morphofunctional reconstruction of the dental-maxillary apparatus affected by edentation has been attempted. sensitive bone immobility, deepening of osteogenesis.

Over the years, studies have been made on the anatomy of edentulous jaws, atrophy and bone resorption processes, nonheterogenesis phenotypes in the use of autogenous, heterogeneous, homogrephous, allograft grafts.

\section{Short history of oral implantology}

In 1915, Congton is the first to use the term "implantation" to describe "the operation of introducing a natural or artificial root into a new alveolus created in the alveolar process."

The evolution of implantology can be structured in six periods:

1. The ancient period - from the beginning to the year $1000 \mathrm{e}$.

2. The medieval period - 1001-1799

3. The foundation period $-1800-1910$

4. The premodern period - $1910-1930$

5. The modern period - $1931-1977$

6. Contemporary oral implantology - 1978-present

\section{The ancient period}

The ancient period is marked by the civilizations of Ancient Egypt and the Middle East. The most used materials for replacing the missing dental structures were animal teeth or ivory scarves.

\section{The medieval period}

In the field of dental transplantation, the Middle Ages is dominated by the name of the Arabian doctor Abul Casis-also known as Abul Casim (963-1013) who expected bull bone transplants. In Japan, in the XV-XVI century, wood anchored in dental roots - the type of early endodontic implant.

In Europe, Ambrosie Pare describes the avatars of the transplants. Pierre Fauchard (1678-1761) and Jhon Hunter (1728-1793) are representative names for the XVIII ${ }^{\text {th }}$ century related to dental transplantation. Since then, there have been criticisms of implants of ivory, bone, metal or human teeth - considered unsatisfactory. However, there is a desire to replace missing teeth by implantation.

\section{The foundation period}

True endosomal implantology begins in the 19th century. Magilio, in 1809, inserted a gold implant into the alveolus of fresh extra teeth. At the end of the 1900s, he rejuvenated natural teeth use as implants. WJYounger of San Francisco is accredited for introducing these interventions into the US. Congdon writes: "Implantation of natural teeth seems to have yielded satisfactory results, and failures were due to resorption or exfoliation.

Younger writes in 1893: "I am happy to note that these operations (implants) have entered the 8th year of life." In 1887, Harris reports in Dental Csmos that he implanted a porcelain tooth into an artificially obtained alveolar. Edmuns of New York reported in 1889 that a metal capsule was implanted in the space occupied by the upper premolar. The capsule is platinum-coated lead and welded with pure silver. Znamenski in 1891 describes the implantation of teeth of porcelain, gutta-percha, and rubber. Bonwell-in 1895 practiced implants of gold or iridium tubes as a support for future coronae.

At the meeting of the National Dental Association in 1898, R.E.Payne is the one who for the first time presents a clinical case: The implant of a silver capsule.

*Correspondence to: Vîrbănescu Cristina Andreea, Faculty of dental medicine, University titu maiorescu, Bucharest, Romania, E-mail: drandreeavi@gmail.com

Received: November 14, 2018; Accepted: November 23, 2018; Published: November 26, 2018 


\section{The premodern period}

R.E.Payne and E.J.Greenfild dominate the first two decades of the XIX century in the implantology issue. Payne presents his capsule implant at the $3^{\text {rd }}$ Dental International Congress.

Greenfild was the first to present a documentation of photographs and diagrams related to an original implant procedure. He put the following question: "If a surgeon can use metals in bone treatment why cannot a dentist use it?" He also emphasizes the importance of asceticism in dental surgery. John Roberts, a known surgeon at that time, writes that when Greenfild, describing his operation, talks about sterilization of instruments and local aseptic with bismuth paste, he is no longer a dentist, but a surgeon.

Burton Lee-Thorpe writes: "I examined a patient of Dr. Greenfild in whose mouth he implanted an artificial root 18 months ago. The root is firmly fixed in the jaw and the gingival tissue is perfectly healthy around the cervical margin." Tomkins in 1925 implanted porcelain teeth.

Brill (1936) introduced rubber rods into an artificially created alveolus. Adams (1937) patented a screw-shaped cylindrical submerged implant having a smooth gingival sleeve and a high rounded retention element.

\section{The modern period}

The modern era of implantology certainly begins at the end of the 1930s with the works of Venable, Struck, and Goldenberg. In 1937, Venable perfected Cr-Co-Mo alloy today known as vitalium. This alloy makes possible the appearance of the new type of implant during the next. In this period, we have:

\section{Stage I endosomal implants}

In 1939, Alvin and Moses Struck pictured the screw-type dental implant from vitalium. Struck perfected transdental and endosal implants, implants appreciated as satisfactory. Strock initiates animal experimental studies to test the tissue response to implants inserted into the jaw in the dog. It presents the histological evidence of a possible "bone congruence" at the implant level after a certain period of operation. This seems to be the first evidence of osteointegration or bone deposition.

\section{The subperiosteal implant}

Evolution of the subperiosteal implant begins with the communication of Dahl's patent in 1942.

Gershkoff and Goldenberg visit Dahl in Sweden and brings to the U.S. the concept of the subperiosteal implant. Initially, the subperiosteal implant was made without direct fingerprinting of the recipient's bone. Isaiah Lew (1951) is the author of the direct bone fingerprinting technique of executing the two-stroke procedure for the subperiosteal implant.

The direct bone fingerprinting method was also experimented by Berman and Marziani. The subperiosteal implant propulsion includes the unilateral subperiosteal implant with lingual flap and Bodin's (1950) implant that implants the alveolar ridge.

E. Popa, after a 25-year experience (1957-1977-over 200 cases) in the subperiosteal implant field, develops the intracortical implant technique, which has enjoyed success and which today, in the light of new research on osteointegration and technology of the implant, is relaunched in a new variant. Linkow proposed tripodal as a subdivision of the subperiosteal implant with the indication in cases of bone resorption marked on the mandible in the area of premolars and molars. The implant is made of titanium by casting.

\section{Stage II endosomal implantation}

New forms of endosomal implants developed between 1940 and 1960.

Formiggini, in 1947, laid the foundation for the simple spiral helical implant, made of stainless steel or tantalum.

Zepponi, a collaborator of Formiggini, perfected the implant, originally executed manually, reaching the cast implant.

The period of the 50-60s was a period of experimentation in the development of new implant types. L. Linkow developed the propi-vent implant plant in 1963.

This implant has similarities with the 1913 Greenfild capsule implant and the contemporary implant Core-vent and Swiss Hollow Basketball. Linkow is considered the most important author in the development of the blade implant, which dominated the 60 s and early 80 s.

The Staple Implant, proposed by Small and Misick, is originally experimented with animals (1966-1967) and applied in the clinic between 1968 and 1973. In 1986, they show 16 years of observation on this type of implant. Kawahara in 1970 experimented with the ceramic implant, in Japan.

The ITI Group in Switzerland is working on the Swiss Hollow Basket implant. Thus, the sphere of oral implantology is expanding, and the process continues in the present.

\section{Contemporary period}

Contemporary oral implantology begins in 1978 when the American Health Institute Conference was held at Harward.

This conference was about oral implantology with appropriate assessments.

In 1980, three factors determine the continued development of oral implantology:

\section{The results of the Harward Conference 1978}

2. The scientific credibility of the studies in Goteborg

3. Extension of scientific research in the field of implantology.

IUT Swiss Basket of Sutter and colleagues from the Straumann Institute is the result of research that began in 1974 on the basis of Greenfild's structural concept.

A. Kirsh designed the IMZ implant in 1974, his cylindrical implant being used in Germany in 1978.

In early 1970, Kawahara, after animal experimental studies, designed the unique ceramic cylindrical implant of aluminum oxide crystal. After the clinical experiments, the implant is introduced in the USA, first by Jhonson \& Jhonson and then by Kyocera Corporation (1980), which has sponsored extensive animal experiments as well as clinical investigations. Most of the implants have been designed according to the Branemark 2-implant model.

The Core-vent implant, a two-stage basket implant was introduced by Nieznik in 1982 .

Nobelpharma implant cylindrical implants like Sterioss, Flexiroot, Osseodent, Screw-vent, Swede-vent were introduced after 1982. 
In 1972, ADA takes a cautious approach to the dental implant.

The report of this association points out that "there is an obviously limited acceptance of dental implantology as a profession in this being a unanimous international point of view. "

After consulting the specialized literature ADA concludes: "Dental implantology has progressed over the last 20 years and has gained ground on many levels."

The dental implantology will only be clear when systematic experiments and future reports will have a unitary conception.

Fundamental and truly scientific research has been almost nonexistent until 1972.

In 1973, the Council for Dental Materials and Devices, together with the National Institute of Dental Research, organized a symposium on "Dental Implants". Based on the report from this Symposium and the one cited above, the ADA recommends in 1974 that "endosomal dental implants may be considered as being in a new phase and need a sustained scientific research." "The endosomal implant is not currently indicated as a routine technique in medical practice."

In 1980 and 1981, the ADA selected the criteria for provisional acceptance of the dental implant project.

The extended acceptance of dental materials, instruments, and equipment for the endoscope implant. The benefits and risks were weighed, the endoscope implanting the field. implant and the patient's complete information on the positivity of the occurrence of failures and risks.

Following the assessment of scientific research, the Dental Materials, Instruments and Equipment report states: "Scientific research is still needed, and the implant is not recommended as a routine method in clinical practice." The Council initiated in 1976 national centralization of implant data.

Data from 93 practitioners, related to 1885 implants, carried out by 1987 were obtained. The findings showed that "the results were in line with the position of the council and that further scientific research is necessary. "Since 1987, three more types of implants have obtained the provisional approval of the Dental and Devize Council: InterporeIMZ in 1988; Oratronix Blade in 1989; Core-vent in 1989. At the ADA conference in 1990, after the debates, based on the accumulated data, both blade and cylindrical submerged implants were accepted.

For the coming years, it has been decided that any endosomal implant introduced into the U.S. should be declared at a computerized data storage center in order to favor a statistical evaluation thereof.

The history of dental implants is further written.

At the beginning of the modern period of oral implantology, only patients with adequate bone volume were candidates for implantprothetic oral rehabilitation. Most of the time, however, patients editing partially or totally requiring implanto-prosthetic treatment to restore both functional and esthetic apparatus dento-maxillary, suffer from important bone atrophy.

Thus, in order to benefit from dental implants, it is necessary to increase the volume of the bruising by using various types of additions and materials.

Although guided bone regeneration techniques have become a routine in oral implantology, bone pre-implant reconstruction is a medical challenge that wants to restore the correct anatomical, functional and aesthetic restoration of masticatory function.

Bone addition techniques combined with correct implant placement can provide the physician and patient with the satisfaction of a successful and esthetic treatment.

\section{Anatomy and bone physiology}

\section{Topic anatomy notions}

The basics required to insert dental implants or to perform safe bone regeneration therapies are given by a thorough knowledge of the topographic anatomy.

Knowledge of anatomy is also necessary to understand complications that may occur accidentally during surgery, such as damage to blood vessels or nerves as well as post-operative complications such as edema or infection.

The bone substrate is represented by the two upper and lower maxillaries in which the teeth are implanted.

The maxillary is a fixed pair with a spongy structure and has a series of cavities that they define - sinuses or participate in their contouring (orbit, nasal fossa). It is placed in the center of the facial massif, on the soft sides of the cheeks, being made up of a body and four processes or apophyses.

The jaw body contains the maxillary sinus and schematically, geometrically, is similar to a pyramidal body with the following four faces:

- The anterior face corresponding to the soft cheeks

- The posterior face defining the pterigo-palatine fossa and the infratemporal fossa. On this face, there is the jaw tuberosity, which has many vertical and horizontal alveolar bastions. The lower part of the tuberosity corresponds to the posterior wall of the upper molar upper alveolus.

- The internal face, oriented to the nasal fossa, participates in the formation of their external wall.

- The upper face, corresponds to the cavity of the orbit, forms the lower wall thereof.

Each maxillary bone is characterized by the existence of four lamellar extensions, known as apophyses: - frontal apophysis is prominently oriented towards the medial wall of the orbit, articulates with the frontal bone. - Zygomatic apophysis is the prominence by which the maxillary bone articulates with the zygomatic bone

The alveolar apophysis is the inferior extension with a horizontal direction in which the dental alveoli are located.

The palatine epiphysis is a bone blade with the same horizontal direction but situated on another plane, it participates in the formation of pale veins in the two previous thirds.

With the loss of teeth, the dental alveoli disappear, and they suffer a bone reshaping process and the nearby gums will cover the remaining defect.

\section{The maxillary sinus}

The anatomy of the maxillary sinus was first described and illustrated by Leonardo da Vinci in 1489, later documented and presented by the English anatomist Nathaniel Highmore in 1651, the 
maxillary sinus known in the modern age as the Highmore Antrum. The maxillary sinus is a pyramidal cavity located deep in the body of the maxillary bone, the largest sinus paranasal. The base of the pyramid is directed to the lateral nasal wall and most often measures $33 \times 33 \mathrm{~mm}$ and the tip extends to the zygomatic bone another $23 \mathrm{~mm}$.

Its dimensions are very different: small, medium or large. Generally, the volume of the sinus cavity is $15 \mathrm{~cm} 3$ per dental adult and its dimensions can vary between $9-20 \mathrm{~cm}$ cubic.

The floor of the maxillary sinus is backed by bone or membranous septums transiting obliquely or transversally from the medial or lateral wall, sometimes dividing the sinus into separate cavities. These elements are present from the canine area to the molar area, Misch observing their tendency to disappear with the age of the edifice to the patient when the stress to the bone is lower.

\section{Mandible}

The mandibula is an arcuate, multicellular, movable bone with a compact structure contributing to face relief, being located in the anterior-inferior part of the visceral cranium. It is the largest and most resilient of skull bones.

The mandible consists of a body and two branches. The mandibular body has a horseshoe shape with a concave rearward orientation.

The body has a lower basal and an upper alveolar, two-sided, external and internal side.

The external face has a convex aspect and is characterized by the following morpho pathological elements: mental protruding, previously located on the midline.

The mental gesture, the place where the vessels and mental nerves come out, is located laterally between the premolars. - the external oblique crest, which is inserted into the orofacial muscles The internal face has a concave appearance and is characterized by the following morphopathological elements: -the inner oblique crest or the milohioidian line, the area on which the milohioidian muscle was inserted '; - the sublingual gland, located above the internal oblique ridge; - the submandibular gland, previously located on the median line

The upper mandible body is the alveolar bone where the lower teeth are located.

The mandible branches sleep with the body, an angle called the gnoniom, on whose external face is the masseteric tuberosity on which the masseter muscle is inserted, and on the inner face is inserted the internal pterihoidian muscle.

The base of the mandible has a compact structure, a predominant element for providing resistance, in cross-section is rounded.

\section{Elements of morphology and physiology of bone tissue}

From a physical point of view, bone tissue has two characteristics: hardness and resistance to deformation and fracture.

Bone tissue is the only tissue in the body that can be cured without any structural or functional deterioration without scarring.

The bone tissue of the healthy adult consists of two types of bone: cortical or compact, forming the long bones diaphysis, the layer of the epiphyses and the internal and external lamellae of the bones and the spongy bone formed by bones called trabeculae of one or more lamellae, bone lamellae, delimiting cavities of different shapes and sizes, creating the appearance of a sponge.
The compact bone is covered and protected by the brush consisting of collagen fibers, osteoblasts, and osteoclasts, the latter providing remodeling, resorption and formation of the bone around the implant. Calcified connective tissue, in the structure of which two components are present: organic and inorganic.

The organ component consists of bone cells and a fundamental substance. $35 \%$ of the total weight of the bone.

The organic component provides the adhesive between the implant and the bone.

The inorganic mineral component is $65 \%$ of the total bone mass and consists of hydroxyapatite, tricalcium phosphate in the form of microcrystals, calcium carbonate, magnesium carbonate, sodium carbonate, citrate, magnesium, sodium, flora. the bone component has stiffness, strength, and weight.

Celulating systems involved in bone formation: Osteoclasts originated in the hematopoietic system and osteoblasts, osteocytes, chondroblasts, chondrocytes - originated in the stromal fibroblast system. Osteoblasts are bone-forming cells originating in mesenchymal stem cells. Osteocytes are formed in after osteoblasts are deposited by the mineralized osteoid. They are long-life cells ( 35 in humans). Osteoclasts are large, syncytial cell formations that play a role in bone modeling.

\section{Osteolysis}

The bone is permanently formed by osteoblasts and is continuously lysed where osteoclasts are active. Osteoblasts are also found on the outer surfaces of the bones and in the bone cavities. Some osteoblastic activity is carried out in all living bones. Osteolysis takes place in the immediate vicinity of osteoclasts.

They emit similar villosities to the bone and secrete two types of substances: proteolytic enzymes and acidic acids, including citric acid and lactic acid. The enzymes digest or disrupt the organic matrix of the bone and the acids solubilize the mineral salts. The balance between osteogenesis and osteolysis: Normally, with the exception of growing bones, osteogenesis, and osteolysis rates are equal so that the total bone mass stays constant.

Osteoclasts exist in the form of small populations that begin to develop by consuming bone, basically digging a tunnel in 3 weeks which can be $1 \mathrm{~mm}$ in diameter and a length of several $\mathrm{mm}$. At the end of this period, osteoclasts stop their function and the tunnel is invaded by osteoblasts. Then the osteogenesis follows, for a few months, the new bone is deposited in successive layers on the internal surface of the cavity until the tunnel is filled.

Osteogenesis ceases when the bone begins to disturb the vessels that irrigate the area. The channel through which these vessels pass, called the Hawersian channel, is so all that remains in the initial cavity. Each new bony territory formed in this way is called osteon. The formation and bone lysis continue with several important physiological functions:

a) The bone adjusts its resistance, usually proportional to its degree of strain. The bones, therefore, change their volume when subjected to high loads.

b) The shape of the bone can be remodeled to adequately support mechanical forces, osteolysis, and osteogenesis, according to the model of the tasks to which it is subjected.

c) As the old organic matrix degenerates, a new organic matrix is needed, thereby maintaining the normal bone consistency. 
The bone is formed in relation to the compressive stress that it has to bear. Continuous physical demand stimulates the osteoblastic deposition of the bone. It is assumed that the osteogenesis at the compressive stress points is determined by a piezoelectric effect, thus: the compression of the bone produces a negative potential at the site compression and a positive potential elsewhere in the bone.

\section{Types of addition materials}

For a successful implantology, the quality and quantity of the saliva and gum should be satisfactory. Tooth loss affects the alveolar process, thus requiring bone addition techniques to achieve optimal results, techniques accompanied by the use of various additive materials. Characteristics of the additive materials:

- Biocompatibility
- osteogenic
- osteoinductive
- Osteoconductive
- Easily manipulable
- Sterile
- Non-Cancerian
- Non-Teratogenic
- Non-Antigene - hydrophilic
- Easily available

The additional materials and the mechanisms by which they act are not all the same, in oral implantology the following types of additive materials are used:

Autograph (autologous) - wounded surgical wound from one area of the body to another area to the same person as -tuberosity -menton -the internal oblique line -the iliac crest -tibia -calvaria

Allograft-tissue transplanted from one person to another

Xenograft-tissue transplanted from animal to person

Synthetic-synthetic materials made in the laboratory

Biological membranes

Accelerators of bone restoration

Bone Graft

It has long been considered as the gold standard in addition techniques, being the only additive material with osteogenic capacities. It does not involve high costs being harvested from the same patient who would receive the graft. It was first in add-on type bone as it was biocompatible with a lower risk than the graft is not acceptable because it comes from the same patient. The incidence of autologous bone graft use was represented by the high rate of intra and postoperative complications as well as high bone loss.

The autograft also contributes to bone growth through BMP growth factors (morphogenetic bone protein) that were released during fixation of the graft to the receptor site with significant osteoinductive qualities. The number of osteoclasts present in the spongy bone is higher than of those present in the cortical bone.

Once harvested, the autograft should be used immediately or stored for short periods of time in sterile saline, Ringier dairy solution or
D5W to maintain the vitality of bone cells. It is contraindicated to keep the grafts in distilled water because cell lysate is produced due to the hypotonicity of the water. Also, keeping grafts in the blood decreases the survival rate of cells in the graft because the red blood cells eliminate cytotoxic cells that damage the cells.

\section{The bone growth mechanism through the use of autographs includes 3 phases}

1st phase (4 weeks) related to osteogenesis and bone regeneration via survival cells. The second phase ( 2 weeks to 6 months with a peak at 6 weeks) related to osteoinduction. The tertiary phase linked to osteoinduction when the bone graft transverse into native bone. Some of the transplanted osteoblasts have a lifetime of 3-4 days with the help of the wound supplied by the vascular tissue surrounding the graft.

Osteoblasts and surviving stem cells from the spongy or cortical bone at the surface of the graft are responsible for the proliferation and formation of the new bone. Blood vessels may grow in a grafted area at the same rate as fibrous tissue increases, $1 \mathrm{~mm}$ per day. Grafting success depends a lot on early and early bone graft vasculature.

The autograph collection places are divided into two categories:

\section{a) Intraoral \\ b) extraoral}

\section{Harvesting from the Memphis mesoniere area}

It is preferred by practitioners due to the triangular shape, slightly curved to the middle, because this form of the harvested graft helps the arched reconstruction of the jaw in the anterior area. The average interforinal emergence is greater than $4 \mathrm{~cm}$ so that larger areas bone atrophy (defects that lie on 3-4 teeth).

The mandibular bone thickness at this level is between 5 and 15 $\mathrm{mm}$.

Pay attention to the vestibular plate not to be fractured, so the thickness of the harvested graft should be less than $2-3 \mathrm{~mm}$ from the total thickness of the mandible. The average mandibular size is $44 \mathrm{~mm}$. The vertebral bone size in this region varies between 5 and $20 \mathrm{~mm}$, and as a general rule graft harvesting will be done taking into account the $5 \mathrm{~mm}$ lengthening from the tops of the anterior mandibular teeth to the base of the mandible. Harvesting from the mandibular branch. This difficulty or anatomy of the patient can often aggravate the harvest in this area. When the 3 molars are missing, it is easy to get large quantities of bone from this place $(3-6 \mathrm{~mm})$.

Copyright: (C)2018 Vîrbănescu CA. This is an open-access article distributed under the terms of the Creative Commons Attribution License, which permits unrestricted use, distribution, and reproduction in any medium, provided the original author and source are credited. 